\title{
Protective effect of active perfusion in porcine models of acute myocardial ischemia
}

\author{
ZANXIANG FENG ${ }^{1}$, ZHIFU MAO ${ }^{1}$, SHENGJUN DONG $^{2}$ and BAOHUI LIU ${ }^{2}$ \\ ${ }^{1}$ Department of Cardiovascular Surgery, Renmin Hospital of Wuhan University, Wuhan, Hubei 430060; \\ ${ }^{2}$ Department of Cardiac Surgery, Binzhou Medical University Hospital, Binzhou, Shandong 256600, P.R. China
}

Received January 1, 2015; Accepted December 8, 2015

DOI: $10.3892 / \mathrm{mmr} .2016 .5665$

\begin{abstract}
Mortality rates associated with off-pump coronary artery bypass (CAB) are relatively high, as the majority of patients requiring $\mathrm{CAB}$ are at a high risk for cardiac events. The present study aimed to establish porcine models of acute myocardial ischemia, and evaluate the protective role of shunt and active perfusion. A total of 30 pigs were randomly assigned to five groups, as follows: i) Sham (control); ii) A1 (shunt; stenosis rate, 55\%); iii) A2 (shunt; stenosis rate, $75 \%$ ); iv) B1 (active perfusion; stenosis rate, 55\%); and v) B2 (active perfusion; stenosis rate, $75 \%$ ) groups. Aortic pressure $\left(\mathrm{P}_{0}\right)$, left anterior descending coronary pressure $\left(\mathrm{P}_{1}\right)$, and coronary effective perfusion pressure $\left(\mathrm{P}_{1} / \mathrm{P}_{0}\right)$ were measured. The expression levels of tumor necrosis factor- $\alpha$ (TNF- $\alpha$ ), cardiac troponin (cTnI), creatine kinase-myocardial band (CK-MB), interleukin (IL)-6, IL-10, B-cell lymphoma 2 (Bcl-2), and caspase- 3 were detected using enzyme-linked immunosorbent assay or western blotting. The myocardial apoptosis rate was determined using the terminal deoxynucleotidyl transferase dUTP nick end labeling assay. Ischemia models with stenosis rates of 55 and $75 \%$ were successfully constructed following suturing of the descending artery. Compared with the control, the 55 and $75 \%$ stenosis groups demonstrated significantly decreased $\mathrm{P}_{1} / \mathrm{P}_{0}$, increased expression levels of TNF- $\alpha$, cTnI, CK-MB, IL-6, IL-10 and caspase-3, an increased rate of myocardial apoptosis, and a decreased expression level of anti-apoptotic protein, Bcl-2. At $30 \mathrm{~min}$ following successful establishment of the model (ST segment elevation to $1 \mathrm{~mm}$ ), group $\mathrm{B}$ demonstrated significantly increased $\mathrm{P}_{1} / \mathrm{P}_{0}$, decreased expression levels of TNF- $\alpha$, cTnI, CK-MB, IL-6, IL-10 and caspase-3, a decreased rate of myocardial apoptosis, and an increased expression level of anti-apoptotic protein, Bcl-2. Furthermore, the current study indicated that active perfusion
\end{abstract}

Correspondence to: Dr Zhifu Mao, Department of Cardiovascular Surgery, Renmin Hospital of Wuhan University, 238 Jiefang Road, Wuchang, Wuhan, Hubei 430060, P.R. China

E-mail: zhifumao011219491949@163.com

Key words: acute myocardial ischemia, animal model, active perfusion, shunt perfusion was more efficacious in maintaining myocardial perfusion and alleviating ischemic injury when compared with traditional shunt perfusion.

\section{Introduction}

Coronary artery disease (CAD) is a leading cause of mortality and morbidity in developed and developing countries (1). An estimated 16.3 million Americans aged $\geq 20$ years have been diagnosed with CAD, and the overall CAD prevalence is $7 \%$ in adults in the US (2). Typically, patients with CAD receive advice for managing the risk factors associated with the progression of atherosclerosis, as well as pharmacological treatment. In addition, some patients may undergo coronary revascularization, which falls into two main categories: Coronary artery bypass grafting (CABG) and catheter-based percutaneous coronary intervention (PCI) (3.4). A number of disadvantages are associated with PCI, including early restenosis and an inability to relieve the totally occluded arteries or vessels that occur with extensive atherosclerotic disease (5). CABG has the advantages of a greater durability (graft patency rates exceed $90 \%$ at 10 years with arterial conduits) and more complete revascularization regardless of the morphology of the obstructing atherosclerotic lesion (6).

Numerous surgeons and centers have adopted off-pump coronary artery bypass (OPCAB). An interest in off-pump techniques has been driven by an increased awareness of the deleterious effects of cardiopulmonary bypass and aortic manipulation (7,8). At present, OPCAB is the first choice for revascularization in patients with severe coronary artery disease; however, the mortality rate associated with this type of surgery is relatively high, since the majority of patients requiring $\mathrm{CAB}$ are at a high risk for cardiac events (9). The intracoronary shunts are designed to maintain myocardial perfusion by maintaining blood supply in the distal myocardium (10). However, as the shunt itself does not increase coronary blood flow, the distal flow remains limited by stenosis, markedly so in severe cases (11). The present study aimed to establish porcine models of myocardial ischemia with different levels of stenosis, and provide myocardial protection using active perfusion at a site distal to the anastomosis. By comparing this to the traditional method of shunt perfusion, the present study investigated the protective effect of this novel perfusion strategy in pigs with acute myocardial infarction. 


\section{Materials and methods}

Animals. A total of 30 male pigs (weight, $50-60 \mathrm{~kg}$ ) were included in the present study. The animals were provided by the Animal Center of Binzhou Medical College (Binzhou, China), where they were maintained under a 12 -h light/dark cycle. The present study was conducted in accordance with the 'Guiding Principles in the Care and Use of Laboratory Animals', as outlined by the Animal Center of Binzhou Medical College, and with approval from the Animal Care Committee of Binzhou Medical College.

Agents. The fentanyl and ketamine hydrochloride injections were purchased from Yichang Humanwell Pharmaceutical Co., Ltd. (Yichang, China). The malondialdehyde enzyme-linked immunosorbent assay (ELISA) kit was purchased from R\&D Systems, Inc. (Minneapolis, MN, USA). The tumor necrosis factor- $\alpha$ (TNF- $\alpha$ ), interleukin (IL)-6 and IL-10 ELISA kits were purchased from Jiancheng Bioengineering Research Institute (Nanjing, China). The cardiac troponin (cTnI), creatine kinase and myoglobin $(\mathrm{Mb})$ ELISA kits were purchased from Beyotime Institute of Biotechnology (Haimen, China). The terminal deoxynucleotidyl-transferase dUTP nick end-labeling (TUNEL) kit was purchased from Roche Diagnostics GmbH (Mannheim, Germany). Atropine, propofol, heparin and lysis buffer were obtained from Sigma-Aldrich (St. Louis, MO, USA). Sodium dodecyl sulfate buffer, polyacrylamide gels, nitrocellulose membranes, Tris-buffered saline containing Tween-20 (TBST), enhanced chemiluminescence (ECL) solution, 4\% paraformaldehyde, paraffin, Triton X-100, phosphate-buffered saline (PBS) and the Bicinchoninic Acid Protein Assay kit were purchased from Kangchen Bio-tech, Inc. (Shanghai, China). Potassium chloride (KCl) was obtained from Sinopharm Chemical Reagent Co., Ltd. (Shanghai, China)

Instruments. The current study used an ALCBio ALC-V10B ventilator (Shanghai Alcott Biotech, Ltd., Shanghai, China), an invasive pressure monitor (BeneView T8; Agilent Technologies, Inc., Santa Clara, CA, USA), an invasive pressure sensor (PT161103; Shenzhen Mindray Bio-Medical Electronics Co., Ltd., Shenzhen, China), a blood pressure monitor (MC-6800; Shenzhen Mindray Bio-Medical Electronics Co., Ltd.), a fluorescence microscope (BX61; Olympus Corporation, Tokyo, Japan), and the SpectraMax ${ }^{\circledR} 190$ Microplate Reader (Molecular Devices, LLC, Sunnyvale, CA, USA).

Active perfusion cannula. A scalp acupuncture hose was used to produce the cannula for active perfusion. The ends of the hose were heated and stretched to thin the wall, until the diameter of the hose reached $\sim 1.5 \mathrm{~mm}$. A ramp cutoff was made at the end of the hose, which was then connected to a T-tube. The ends of the T-tube were connected to a $16 \mathrm{G}$ syringe (inner diameter, $1.19 \mathrm{~mm}$ ), for perfusion, and a blood pressure monitor.

Grouping of animals. Following 2 days of adaptive feeding, where the pigs were given free access to food and water and were housed apart and maintained under a 12-h light/dark cycle, the pigs were randomly assigned to five groups $(n=6)$, as follows: i) Sham (control); ii) A1 (shunt; stenosis rate, 55\%); iii) A2 (shunt; stenosis rate, 75\%); iv) B1 (active perfusion; stenosis rate, 55\%); and v) B2 (active perfusion; stenosis rate, $75 \%)$.

Establishment of porcine models of acute stenosis. Sedation was provided by intramuscular injection of atropine ( $3 \mathrm{mg}$ ). Ketamine $(0.1 \mathrm{mg})$ and fentanyl $(0.3 \mathrm{~g})$ were administered 5 min after atropine injection. The pigs were placed in the supine position with their limbs fixed on the operating table, and underwent endotracheal intubation using fentanyl and propofol $(6 \mathrm{mg} / \mathrm{kg} / \mathrm{h})$ for anesthesia. Following preparation of the skin and monitoring by electrocardiogram (ECG), a midline incision was made in the chest $(3 \mathrm{mg} / \mathrm{kg}$ heparin for heparinization), and a pressure sensor was placed in the ascending aorta for measuring aortic pressure $\left(\mathrm{P}_{0}\right)$. The proximal left anterior descending artery $(\sim 1 \mathrm{~cm})$ was freed and transiently clipped to allow measurement of the lumen diameter. In the control group, the lumen was opened following occlusion, however, for the experimental group, the suture was positioned using a 7-0 Prolene thread. The suture was made at a length of one-third and one-half of the lumen diameter, thus the remaining size of the lumen was four-ninths and one-quarter of the original, thereby achieving a stenosis rate of 55 and $75 \%$, respectively. A trocar was punctured in the distal third of the descending branch, and was then connected to the pressure sensor to monitor the left anterior descending coronary pressure $\left(\mathrm{P}_{1}\right)$, in order to calculate the effective perfusion pressure $\left(\mathrm{P}_{1} / \mathrm{P}_{0}\right)$.

Myocardial perfusion strategy. In the sham group, $\mathrm{P}_{0}$ and left anterior descending coronary pressure $\left(\mathrm{P}_{1}\right)$ were monitored at 30 min following opening of the coronary artery. In group A, an incision was made on the coronary artery to insert a shunt when the ST segment elevation reached $1 \mathrm{~mm} . \mathrm{P}_{0}$ and $\mathrm{P}_{1}$ were monitored following insertion of the shunt, and a blood sample $(5 \mathrm{ml})$ was taken at $30 \mathrm{~min}$ post-perfusion. In group B, an active perfusion needle was pierced into the ascending aorta when ST segment elevation reached $1 \mathrm{~mm}$, and $\mathrm{P}_{0}$ was monitored using the T-tube. An incision was made on the coronary artery to insert the active perfusion tube distal to the vessel, and active perfusion was initiated by opening the tube. $\mathrm{P}_{1}$ was monitored during active perfusion, and a blood sample $(5 \mathrm{ml})$ was collected at 30 min post-perfusion.

Following blood sampling, the superior and inferior vena cava and the ascending aorta were clipped, and the heart was emptied via the aortic root. The pigs were sacrificed by intravenous injection with $10 \% \mathrm{KCl}$ immediately following blood emptying, and the apical myocardium at the left ventricle was sampled $(10 \mathrm{~g})$ for further examination.

Determination of serum $T N F-\alpha, c T n I, M b$, creatine kinase-MB (CK-MB), IL- 6 and IL-10 using ELISAs. The ELISA kits were used according to the manufacturer's protocols. Briefly, blood samples $(5 \mathrm{ml})$ were placed in an ethylenediaminetetraacetic acid-containing Eppendorf tube (500 $\mu \mathrm{l})$ to allow thorough mixing for $20 \mathrm{~min}$. The mixture was centrifuged at 2,500 x g for $20 \mathrm{~min}$ to collect the supernatant. The concentration/optical density (OD) value standard curve was generated using the serially diluted standards provided with the kit. An automated microplate reader (SpectraMax ${ }^{\circledR} 190$ ) was used to measure the 

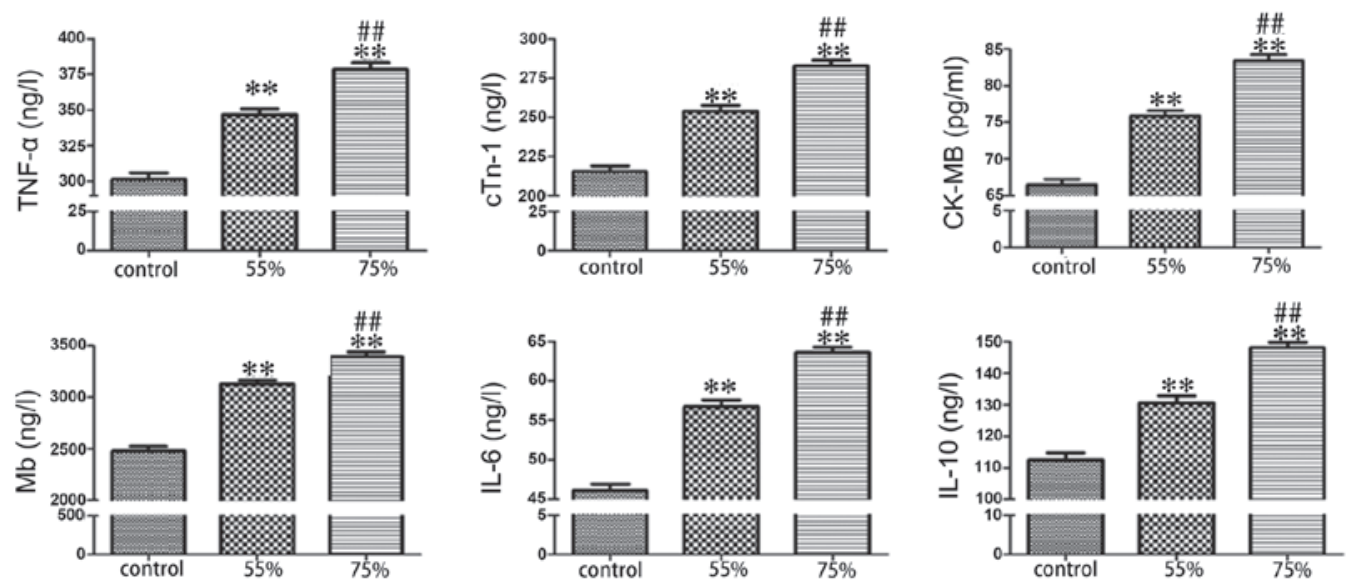

Figure 1. Serum level of TNF- $\alpha$, cTnI, CK-MB, Mb, IL-6 and IL-10 30 min after stenosis formation. ${ }^{* *} \mathrm{P}<0.01$ vs. the control; ${ }^{\# \prime} \mathrm{P}<0.01$ vs. the $55 \%$ stenosis group. TNF- $\alpha$, tumor necrosis factor- $\alpha$; cTnI, cardiac troponin; CK-MB, creatine kinase-myocardial band; Mb, myoglobin; IL, interleukin.

OD value of the centrifuged serum, and the concentration of target proteins was calculated according to their positions on the standard curve.

Western blot analysis of caspase-3 and B-cell lymphoma 2 (Bcl-2) in myocardial tissue samples. The collected myocardial tissue samples were homogenized in a lysis buffer and centrifuged at $12,000 \mathrm{x}$ g for $15 \mathrm{~min}$. The lysates were collected and underwent protein quantification using the bicinchoninic acid assay. A total of $20 \mathrm{~g}$ protein was boiled with $1 \mathrm{X}$ sodium dodecyl sulfate (SDS) buffer for SDS-polyacrylamide gel electrophoresis ( $80 \mathrm{v}$ for $30 \mathrm{~min}$ ), and the electrophoresed proteins were transferred onto a nitrocellulose membrane. Western blotting was performed by blocking the membrane with TBST containing $5 \mathrm{~g} / 1$ skimmed milk for $1.5 \mathrm{~h}$. The membrane was incubated with primary antibodies against caspase-3, Bcl-2 and $\beta$-actin $(1: 1,000$; Cell Signaling Technology, Inc., Danvers, MA, USA) at $4^{\circ} \mathrm{C}$ overnight. The membrane was washed with TBST three times (10 min each time), and re-incubated with horseradish peroxidase-conjugated goat anti-rabbit or goat anti-rat immunoglobulin $\mathrm{G}$ (1:5,000; Zhongshan Bio-tech Co., Ltd., Zhongshan, China) at room temperature for $2 \mathrm{~h}$, followed by washing with TBST three times (10 min each time). Blots were developed using the ECL solution, and Quantity One 4.62 software was used to analyze the expression level of target proteins (Bio-Rad Laboratories, Inc., Hercules, CA, USA).

Measurement of myocardial tissue apoptosis using the terminal deoxynucleotidyl transferase dUTP nick end labeling (TUNEL) assay. The myocardial tissue samples were fixed with $4 \%$ paraformaldehyde for $24 \mathrm{~h}$, and dehydrated and embedded in paraffin. The samples were sliced at a thickness of $0.6 \mathrm{~mm}$, and were perforated for $5 \mathrm{~min}$ with Triton X-100, followed by washing with PBS three times (5 min each time). TUNEL was performed according to the manufacturer's protocols, and all surgical procedures were performed under dark room conditions. The sections were treated with $50 \mu 1$ TUNEL solution (containing $2 \mu 1$ terminal deoxynucleotidyl transferase (TdT) enzyme and $48 \mu$ l fluorescent label) for $60 \mathrm{~min}$ at $37^{\circ} \mathrm{C}$ and were washed with PBS
Table I. Time required for ST segment elevation of $1 \mathrm{~mm}$ following establishment of the model.

\begin{tabular}{lcc}
\hline & \multicolumn{2}{c}{ Stenosis } \\
\cline { 2 - 3 } Parameter & $55 \%$ & $75 \%$ \\
\hline $\mathrm{T}{ }_{\mathrm{ST}}(\mathrm{min})$ & $20.12 \pm 0.74$ & $15.35 \pm 0.66^{\mathrm{a}}$ \\
\hline
\end{tabular}

${ }^{\mathrm{a}} \mathrm{P}<0.01$ vs. the $55 \%$ stenosis group.

Table II. $\mathrm{P}_{1}$ and $\mathrm{P}_{1} / \mathrm{P}_{0}$ ratio following establishment of the stenosis model ( $\mathrm{n}=6$; mean \pm standard deviation).

\begin{tabular}{lccc}
\hline & & \multicolumn{2}{c}{ Stenosis } \\
\cline { 3 - 4 } Pressure & Control & $55 \%$ & $75 \%$ \\
\hline $\mathrm{P}_{0}(\mathrm{mmHg})$ & $108 \pm 6.83$ & $96 \pm 6.26$ & $91 \pm 4.83$ \\
$\mathrm{P}_{1}(\mathrm{mmHg})$ & $106 \pm 5.20$ & $70 \pm 5.34^{\mathrm{a}}$ & $46 \pm 5.11^{\mathrm{b}}$ \\
$\mathrm{P}_{1} / \mathrm{P}_{0}$ & $0.98 \pm 0.03$ & $0.72 \pm 0.04^{\mathrm{a}}$ & $0.51 \pm 0.03^{\mathrm{a}, \mathrm{b}}$ \\
\hline
\end{tabular}

${ }^{\mathrm{a}} \mathrm{P}<0.01$ vs. sham; ${ }^{\mathrm{b}} \mathrm{P}<0.01$ vs. the $55 \%$ stenosis group. $\mathrm{P}_{0}$, aortic pressure; $\mathrm{P}_{1}$, left anterior descending coronary pressure; $\mathrm{P}_{1} / \mathrm{P}_{0}$, coronary effective perfusion pressure.

(three times for $5 \mathrm{~min}$ ) and embedded in paraffin. Green fluorescence was observed under a fluorescence microscope, and 10 fields were randomly selected for cell counting. The rate of apoptosis was calculated as follows: Apoptotic cell count / total cell count $\times 100 \%$. The experiment was repeated three times.

Statistical analysis. Statistical analysis was performed using SPSS 13.0 software (SPSS, Inc., Chicago, IL, USA). Data were presented as the mean \pm standard deviation. Statistical differences between the $55 \%$ and $75 \%$ stenosis groups were assessed using a t one-way analysis of variance. $\mathrm{P}<0.05$ was considered to indicate a statistically significant difference. 
Table III. $\mathrm{P}_{1}$ and $\mathrm{P}_{1} / \mathrm{P}_{0}$ ratio following shunt or active perfusion.

\begin{tabular}{lrrrr}
\hline Pressure & \multicolumn{1}{c}{ A1 } & A2 & B1 & B2 \\
\hline $\mathrm{P}_{0}(\mathrm{mmHg})$ & $85 \pm 6.26$ & $81 \pm 4.83$ & $87 \pm 4.43$ & $82 \pm 4.88$ \\
$\mathrm{P}_{1}(\mathrm{mmHg})$ & $59 \pm 5.34$ & $40 \pm 5.11$ & $69 \pm 4.81^{\mathrm{a}}$ & $65 \pm 5.31^{\mathrm{b}}$ \\
$\mathrm{P}_{1} / \mathrm{P}_{0}$ & $0.70 \pm 0.04$ & $0.51 \pm 0.04$ & $0.81 \pm 0.04^{\mathrm{a}}$ & $0.80 \pm 0.03^{\mathrm{b}}$ \\
\hline
\end{tabular}

Data are presented as the mean \pm standard deviation $(\mathrm{n}=6)$. ${ }^{\mathrm{a}} \mathrm{P}<0.01 \mathrm{vs}$. $\mathrm{A} 1 ;{ }^{\mathrm{b}} \mathrm{P}<0.01 \mathrm{vs}$. A2. $\mathrm{P}_{0}$, aortic pressure; $\mathrm{P}_{1}$, left anterior descending coronary pressure; $\mathrm{P}_{1} / \mathrm{P}_{0}$, coronary effective perfusion pressure; $\mathrm{A} 1$ (shunt; stenosis rate, 55\%); $\mathrm{A} 2$ (shunt; stenosis rate, $75 \%$ ); $\mathrm{B} 1$ (active perfusion; stenosis rate, 55\%); B2 (active perfusion; stenosis rate, $75 \%$ ).

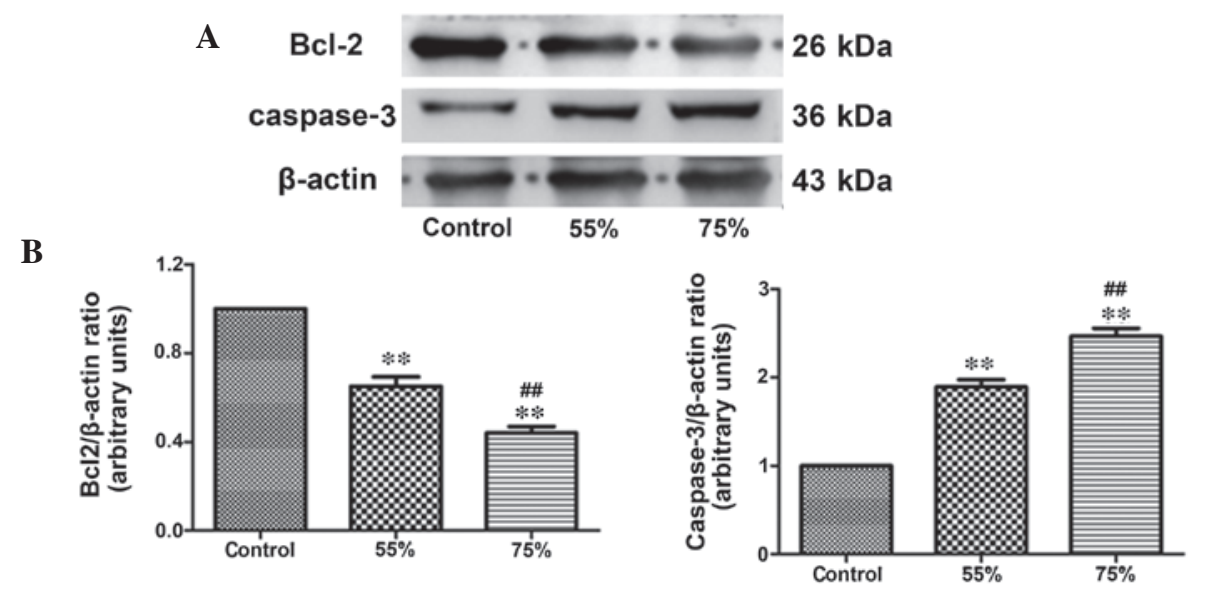

Figure 2. Myocardial expression levels of Bcl-2 and caspase-3 30 min after stenosis formation. (A) Western blots of Bcl-2 and caspase-3; (B) Grey value statistics of Bc1-2 and caspase-3. ${ }^{* *} \mathrm{P}<0.01$ vs. the control; ${ }^{\# \#} \mathrm{P}<0.01$ vs. the $55 \%$ stenosis group. Bcl-2, B-cell lymphoma 2.
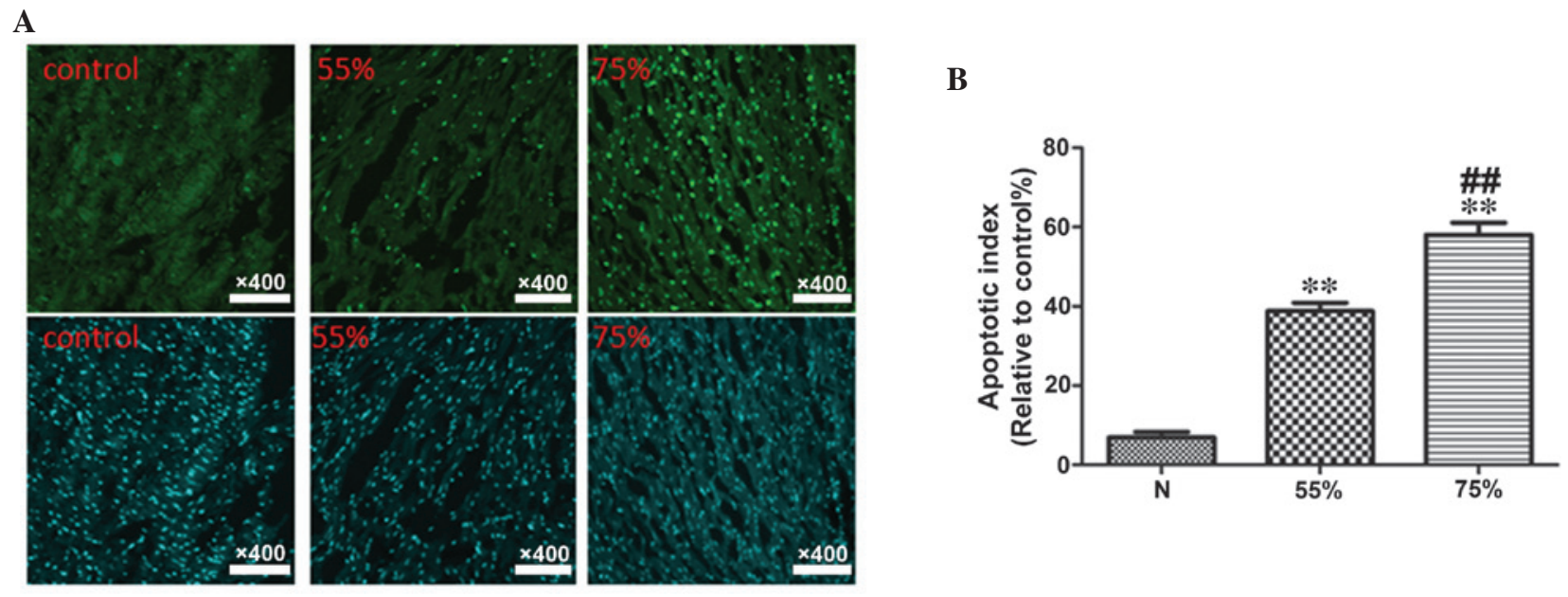

Figure 3. Myocardial apoptosis rate 30 min after stenosis formation. (A) The apoptosis of myocardial cells was assessed by performing a TUNEL assay. TUNEL staining was performed to stain the nuclei of the apoptotic cells (green), and DAPI was used to stain all of the nuclei (blue; magnification, x400; $1 \mathrm{~cm}=100 \mu \mathrm{m})$. (B) Cellular apoptosis is expressed as the apoptotic index, which was calculated using the following formula: Apoptotic index $(\%)=$ the number of positively stained apoptotic cells/the total number of cells counted $\mathrm{x} 100 \%$. ${ }^{* *} \mathrm{P}<0.01$ vs. the control; ${ }^{\# \#} \mathrm{P}<0.01$ vs. the 55\% stenosis group. DAPI, 4',6-diamidino-2-phenylindole; TUNEL, terminal deoxynucleotidyl transferase dUTP nick end labelling; N, control.

\section{Results}

Successful establishment of the model. Pigs in all groups survived the experimental period. Following formation of the stenosis, the ST segment, observed on an ECG, elevated gradually, and elevation of $1 \mathrm{~mm}$ indicated successful construction of the model. The time for establishment of the model shortened with the increase in severity of the stenosis (Table I). Groups with $75 \%$ stenosis exhibited a significantly shortened duration for establishment of the model compared with groups with $55 \%$ stenosis $(\mathrm{P}<0.05)$. 

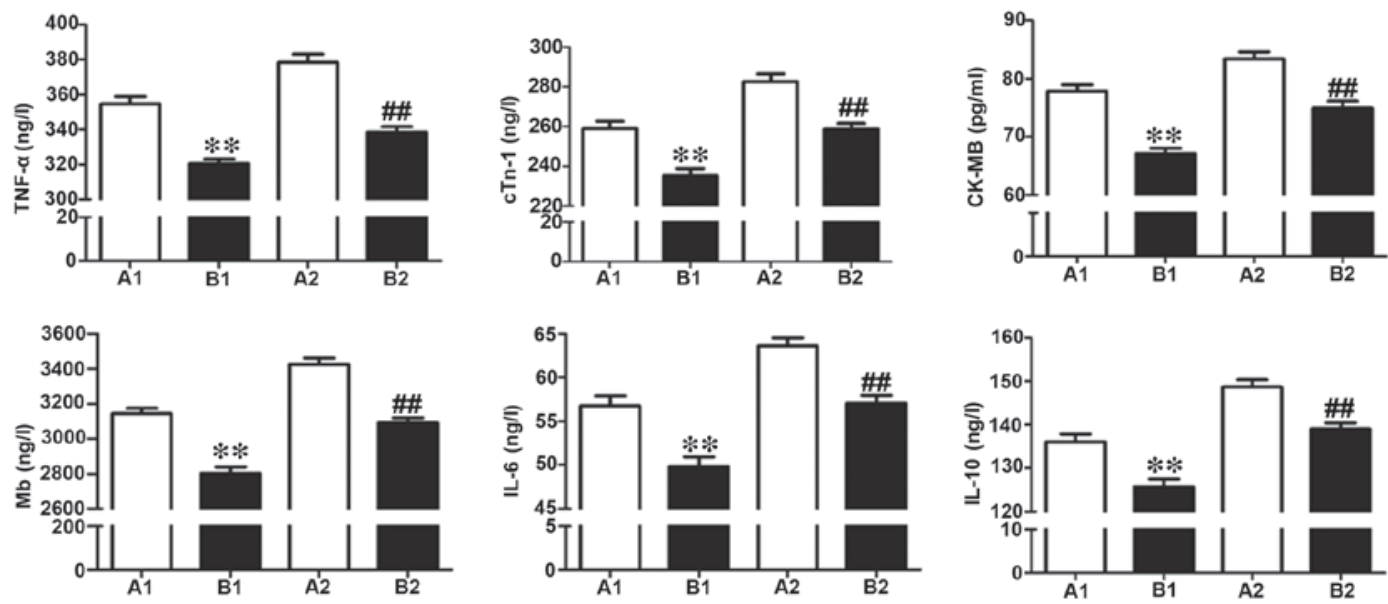

Figure 4. Serum level of TNF- $\alpha$, cTnI, CK-MB, Mb, IL-6 and IL-10 30 min after shunt or active perfusion. ${ }^{* *} \mathrm{P}<0.01$ vs. A1; ${ }^{\# \#} \mathrm{P}<0.01$ vs. A2. TNF- $\alpha$, tumor necrosis factor- $\alpha$; cTnI, cardiac troponin; CK-MB, creatine kinase-myocardial band; Mb, myoglobin; IL, interleukin.
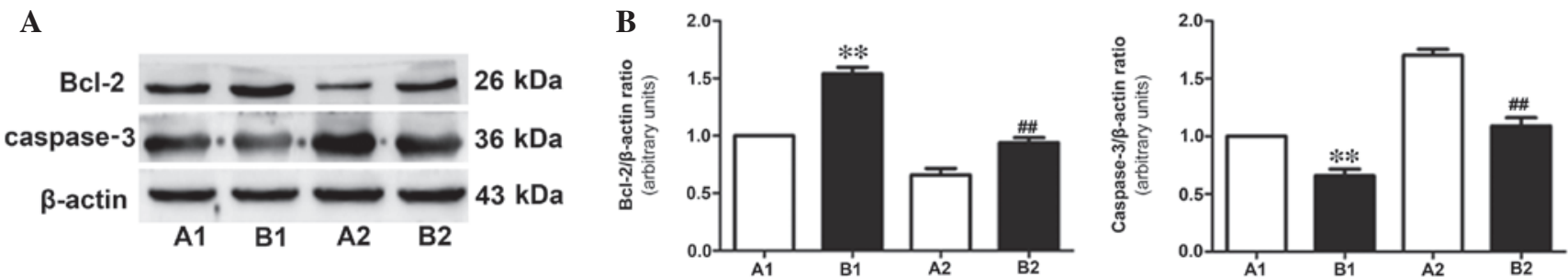

Figure 5. Myocardial expression levels of Bcl-2 and caspase-3 30 min after shunt or active perfusion. (A) The protein expression levels of Bcl-2 and caspase-3 were determined by an immunoblot, in which $\beta$-actin was the protein loading control. (B) The ratios of Bcl-2/ $\beta$-actin and caspase-3/ $\beta$-actin. ${ }^{* *} \mathrm{P}<0.01$ vs. A1; ${ }^{\# \#} \mathrm{P}<0.01$ vs. A2.

A
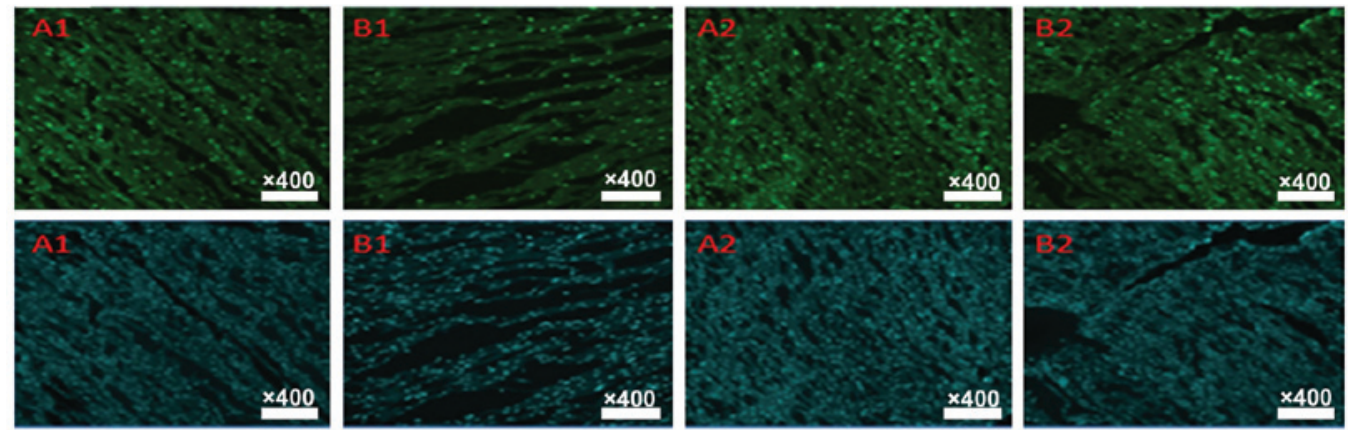

B

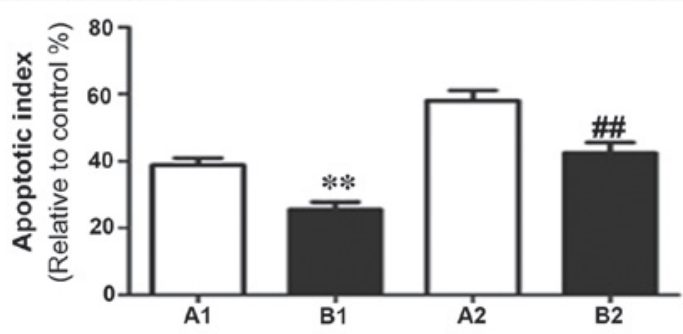

Figure 6. Myocardial apoptosis rate 30 min after shunt or active perfusion. (A) The apoptosis of myocardial cells was assessed by performing a TUNEL assay. TUNEL staining was performed to stain the nuclei of the apoptotic cells (green), and DAPI was used to stain all of the nuclei (blue; magnification, x400; $1 \mathrm{~cm}=100 \mu \mathrm{m})$. (B) Cellular apoptosis is expressed as the apoptotic index, which was calculated using the following formula: Apoptotic index $(\%)=$ the number of positively stained apoptotic cells/the total number of cells counted x $100 \%$. ${ }^{* *} \mathrm{P}<0.01 \mathrm{vs}$. A1; ${ }^{\# \#} \mathrm{P}<0.01$ vs. A2. DAPI, 4',6-diamidino-2-phenylindole; TUNEL, terminal deoxynucleotidyl transferase dUTP nick end labeling.

Models with stenosis developed myocardial ischemia due to the significant decrease in $\mathrm{P}_{1}$, and pigs with a higher rate of stenosis demonstrated lower $\mathrm{P}_{1}$. As presented in Table II, the $\mathrm{P}_{1}$ and $\mathrm{P}_{1} / \mathrm{P}_{0}$ ratio in all experimental groups were significantly 
lower than the control (sham group; $\mathrm{P}<0.01$ ). In addition, the absolute $\mathrm{P}_{1}$ value and the $\mathrm{P}_{1} / \mathrm{P}_{0}$ ratio in pigs with $75 \%$ stenosis were significantly lower compared with the $55 \%$ stenosis groups $(\mathrm{P}<0.01)$.

Following formation of the stenosis, ELISA was used to determine the expression level of serum TNF- $\alpha$, cTnI, CK-MB, Mb, IL-6 and IL-10 (Fig. 1). Western blotting was used to detect the expression level of the apoptotic protein, caspase-3 and the anti-apoptotic protein, Bcl-2 (Fig. 2). In addition, TUNEL staining demonstrated the rate of apoptosis in myocardial tissue samples (Fig. 3). Compared with the sham group, the stenosis groups exhibited significantly increased levels of ischemic and apoptotic indicators, while the level of anti-apoptotic protein, $\mathrm{Bcl}-2$ was decreased $(\mathrm{P}<0.01)$. In addition, the elevation of ischemic and apoptotic indicators, and the decline of Bcl-2 in the $75 \%$ stenosis groups were significant compared with the $55 \%$ stenosis groups $(\mathrm{P}<0.01$; Figs. 1-3).

Comparison of the two perfusion strategies. As the time of ischemia lengthened, the $\mathrm{P}_{1} / \mathrm{P}_{0}$ indicated further decline. Compared with group $\mathrm{A}$, group $\mathrm{B}$ demonstrated significantly elevated $\mathrm{P}_{1}$ and $\mathrm{P}_{1} / \mathrm{P}_{0}$ ratio $(\mathrm{P}<0.05)$, indicating a significant decrease in $\mathrm{P}_{1} / \mathrm{P}_{0}$ in the artery distal to coronary stenosis $(\mathrm{P}<0.01)$. The $\mathrm{P}_{1} / \mathrm{P}_{0}$ in the active perfusion groups $(\mathrm{B} 1$ and $\mathrm{B} 2)$ was higher than in the corresponding shunt groups (A1 and $\mathrm{A} 2 ; \mathrm{P}<0.05$ ), and the difference was more significant in groups with the higher stenosis rate. The $\mathrm{P}_{1} / \mathrm{P}_{0}$ provided by shunt decreased significantly as the severity of stenosis increased $(\mathrm{P}<0.05)$, indicating that blood supply at the distal artery is limited by coronary stenosis. However, as active perfusion was not affected by coronary stenosis, no significant difference in blood supply was detected in the models with different levels of stenosis ( $\mathrm{P}>0.05$; Table III).

Comparison of myocardial injury indicators in the two perfusion groups. Coronary perfusion was performed at 30 min following formation of the stenosis, and ELISA was used to detect the level of myocardial injury indicators, including TNF- $\alpha$, cTnI, CK-MB, Mb, IL-6 and IL-10. Compared to group A, group B demonstrated significantly decreased expression levels of all myocardial injury indicators, indicating the myocardial protective function of active perfusion (Fig. 4).

Comparison of apoptosis-associated proteins, caspase-3 and $\mathrm{Bcl}-2$ in the two perfusion groups. Following $30 \mathrm{~min}$ of coronary perfusion, western blotting was performed to measure the expression levels of anti-apoptotic protein, Bcl-2 and apoptotic protein, caspase-3. As presented in Fig. 5, in group B, the expression level of caspase-3 was downregulated $(\mathrm{P}<0.05)$ while $\mathrm{Bcl}-2$ was upregulated compared with group $\mathrm{A}$, indicating that active perfusion alleviated myocardial ischemia more successfully than conventional shunt perfusion.

Determination of myocardial apoptosis rate using TUNEL. The results of TUNEL staining and the data statistics are presented in Fig. 6. Compared with the corresponding subgroups in group A, group B demonstrated a significantly decreased myocardial apoptosis rate $(\mathrm{P}<0.05)$.

\section{Discussion}

Acute myocardial dysfunction predominantly manifests as ischemic injury (12), and it is particularly prominent under ischemic, function-inhibitory or necrotic conditions (4). Currently, myocardial protections implemented in coronary artery bypass grafting (CABG) include improvement in myocardial perfusion and stress response, as well as alleviation of reperfusion injury. During $\mathrm{CABG}$, the combined use of antegrade, retrograde and bypass perfusion may improve the uneven distribution of cardioplegic fluid (13), and during OPCAB, a shunt alleviates myocardial ischemia by maintaining continuous blood flow at the anastomosis. Ischemic preconditioning includes intraoperative and preoperative preconditioning (14). Therapeutic agents, such as $\beta-1,3 / 1,6$ glucan (15), levosimendan (16), and diazoxide are commonly used in preconditioning, while other studies have suggested that preconditioning with hyperbaric oxygen may also reduce myocardial injury during ischemia-reperfusion $(17,18)$. Therapeutic agents, including atrial natriuretic peptide (19), nesiritide, carvedilol, sodium-hydrogen exchange inhibitor, erythropoietin, reamberin, L-arginine, large doses of insulin, and $\mathrm{C} 1$ esterase inhibitor are often administered to alleviate reperfusion injury (20,21). Despite these measures, myocardial protection during $\mathrm{CAB}$ is unsatisfactory, as myocardial blood supply or preservation fluid perfusion has always been affected and limited by proximal coronary artery stenosis (22).

In conclusion, in the present study, surgical methods were used to establish porcine models of myocardial ischemia with controllable degrees of stenosis at the descending coronary artery, to more effectively simulate the pathological anatomy of coronary heart disease. Active perfusion of the coronary artery was performed distal to stenosis via cannulation across the anastomosis, thus solving the fundamental problem of ischemic cardiomyopathy and providing a more effective method for myocardial protection in clinical CAB. In further studies, hemodynamic methods are required to investigate the distribution and variation of pressure at the coronary artery proximal and distal to stenosis, following formation of the stenosis. On the basis of this, we expect to modify active perfusion strategies, including altering the location of active perfusion at the coronary artery distal to stenosis and the size of the cannula for active perfusion.

\section{Acknowledgements}

The present study was supported by grants from the Academic Promotion Project of Binzhou Medical University Hospital (grant no. BY2011KJ024). The authors would like to thank Dr Yongguang Xiao of Renmin Hospital of Wuhan University (Wuhan, China) for the animals used in the present study.

\section{References}

1. Al Shammeri O, Stafford RS, Alzenaidi A, Al-Hutaly B and Abdulmonem A: Quality of medical management in coronary artery disease. Ann Saudi Med 34: 488-493, 2014.

2. Bashinskaya B, Nahed BV, Walcott BP, Coumans JV and Onuma OK: Socioeconomic status correlates with the prevalence of advanced coronary artery disease in the United States. PLoS One 7: e46314, 2012. 
3. Liu Y, Zhou X, Jiang H, Gao M, Wang L, Shi Y and Gao J: Percutaneous coronary intervention strategies and prognosis for graft lesions following coronary artery bypass grafting. Exp Ther Med 9: 1656-1664, 2015.

4. Deja MA and Malinowski M: Conditioning the heart in cardiac surgery. Kardiol Pol 69 (Suppl 3): S80-S84, 2011 (In Polish).

5. Gorenoi V, Dintsios CM, Schonermark MP, Hagen A: Drugeluting stents vs. coronary artery bypass-grafting in coronary heart disease. GMS Health Technol Assess 4: c13, 2008.

6. Alexander JH and Smith PK: Coronary-artery bypass grafting. N Engl J Med 374: 1954-1964, 2016.

7. Luo T and Ni Y: Short-term and long-term postoperative safety of off-pump versus on-pump coronary artery bypass grafting for coronary heart disease: A meta-analysis for randomized controlled trials. Thorac Cardiovasc Surg 63: 319-327, 2015.

8. Wu S, Wan F, Zhang Z, Zhao H, Cui ZQ and Xie JY: Redo coronary artery bypass grafting: On-pump and off-pump coronary artery bypass grafting revascularization techniques. Chin Med Sci J 30: 28-33, 2015.

9. Gomez-Lara J, Roura G, Blasco-Lucas A, Ortiz D, Sbraga F, Romaguera R, Ferreiro JL, Teruel L, Sanchez-Elvira G, Homs S, et al: Global risk score for choosing the best revascularization strategy in patients with unprotected left main stenosis. J Invasive Cardiol 25: 650-658, 2013.

10. Vallely MP and Ross DE: Intracoronary shunts and off-pump surgery. Ann Thorac Surg 90: 700-701, 2010.

11. Guizilini S, Viceconte M, Esperanca GT, Bolzan DW, Vidotto M, Moreira RS, Câncio AA and Gomes WJ: Pleural subxyphoid drain confers better pulmonary function and clinical outcomes in chronic obstructive pulmonary disease after off-pump coronary artery bypass grafting: A randomized controlled trial. Rev Bras Cir Cardiovasc 29: 588-594, 2014.

12. Wagner R, Piler P, Gabbasov Z, Maruyama J, Maruyama K, Nicovsky $\mathbf{J}$ and Kruzliak P: Adjuvant cardioprotection in cardiac surgery: Update. BioMed Res Int 2014: 808096, 2014.

13. Goncu MT, Sezen M, Toktas F, Ari H, Gunes M, Tiryakioglu O and Yavuz S: Effect of antegrade graft cardioplegia combined with passive graft perfusion in on-pump coronary artery bypass grafting. J Int Med Res 38: 1333-1342, 2010.

14. Teoh LK, Grant R, Hulf JA, Pugsley WB and Yellon DM: The effect of preconditioning (ischemic and pharmacological) on myocardial necrosis following coronary artery bypass graft surgery. Cardiovasc Res 53: 175-180, 2002.
15. Aarsaether E, Rydningen M, Einar Engstad R and Busund R: Cardioprotective effect of pretreatment with beta-glucan in coronary artery bypass grafting. Scand Cardiovasc J 40: 298-304, 2006.

16. Tritapepe L, De Santis V, Vitale D, Santulli M, Morelli A, Nofroni I, Puddu PE, Singer M and Pietropaoli P: Preconditioning effects of levosimendan in coronary artery bypass grafting-a pilot study. Br J Anaesth 96: 694-700, 2006.

17. Yogaratnam JZ, Laden G, Guvendik L, Cowen M, Cale A and Griffin S: Pharmacological preconditioning with hyperbaric oxygen: Can this therapy attenuate myocardial ischemic reperfusion injury and induce myocardial protection via nitric oxide? J Surg Res 149: 155-164, 2008

18. Jeysen ZY, Gerard L, Levant G, Cowen M, Cale A and Griffin S: Research report: The effects of hyperbaric oxygen preconditioning on myocardial biomarkers of cardioprotection in patients having coronary artery bypass graft surgery. Undersea Hyperb Med 38: 175-185, 2011.

19. Sezai A, Hata M, Wakui S, Niino T, Takayama T, Hirayama A, Saito S and Minami K: Efficacy of continuous low-dose hANP administration in patients undergoing emergent coronary artery bypass grafting for acute coronary syndrome. Circ J 71: 1401-1407, 2007.

20. Fattouch K, Bianco G, Speziale G, Sampognaro R, Lavalle C, Guccione F, Dioguardi P and Ruvolo G: Beneficial effects of $\mathrm{C} 1$ esterase inhibitor in ST-elevation myocardial infarction in patients who underwent surgical reperfusion: A randomised double-blind study. Eur J Cardiothorac Surg 32: 326-332, 2007.

21. Sidorenko GI, Gelis LG, Medvedeva EA, Ostrovskiı̌ IuP, Lazareva IV, Sevruk TV, Shibeko NA and Petrov IuP: Pharmacological protection of the myocardium with reamberin in coronary artery bypass grafting in patients with postinfarction angina. Ter Arkh 83: 35-40, 2011 (In Russian).

22. Michelis KC, Boehm M and Kovacic JC: New vessel formation in the context of cardiomyocyte regeneration - the role and importance of an adequate perfusing vasculature. Stem Cell Res 13 (3 Pt B): 666-682, 2014. 